Chirurg 2014 $\cdot 85: 1013$

DOI 10.1007/s00104-014-2904-x

Online publiziert: 12. November 2014

c) Springer-Verlag Berlin Heidelberg 2014

T. Franzke $\cdot$ J. Jähne

Klinik für Allgemein- und Viszeralchirurgie, Schwerpunkt für endokrine und onkologische Chirurgie,

Sektion für Adipositaschirurgie, Diakoniekrankenhaus Henriettenstiftung Hannover

\title{
Lebensqualität nach Gastrektomie und subtotaler distaler Resektion bei Magenkarzinomen
}

\section{Orginalpublikation}

Goh YM, Gillespie C, Couper G, PatersonBrown S (2014) Quality of life after total and subtotal gastrectomy for gastric carcinoma. Surgeon doi:10.1016/j.surge.2014.07.002. (Epub ahead of print)

\section{Hintergrund}

Grundvoraussetzung für eine kurative Resektion bei Magenkarzinomen ist die lokale R0-Situation mit entsprechendem Sicherheitsabstand sowie die Durchführung einer adäquaten D2-Lymphadenktomie. Daher scheint bei entsprechend distal gelegenen Tumoren eine subtotal-distale Resektion (SG) in kurativer Intention möglich zu sein. Befürworter der Gastrektomie (TG) argumentieren jedoch, dass die TG mit einer verhältnismäßig niedrigen Morbidität und Mortalität (MuM) durchgeführt werden kann und gleichzeitig die Gefahr eines proximalen Residualtumors respektive eines späteren intragastralen Rezidivs verhindert wird. Hingegen wird von den Befürwortern einer SG argumentiert, dass die Inkaufnahme einer höheren MuM und einer schlechteren postoperativen Lebensqualität (QOL) bei nicht bewiesenem Überlebensvorteil die Durchführung einer TG nicht rechtfertigt. Die vorliegende Studie widmet sich daher der Frage nach der QOL von Patienten nach TG und SG.

\section{Methodik/Patienten}

Alle noch lebenden Patienten, die zwischen 1994 und 2009 am Royal Infirmary of Edinburgh aufgrund eines Magenkarzinoms operiert wurden, wurden in die Fragebogenstudie einbezogen. Überwiegend wurde nach erfolgter Resektion eine Rekonstruktion nach Y-Roux durchgeführt. Die Entscheidung des Resektionsausmaßes wurde intraoperativ individuell gefällt. Zur Erhebung der QOL, postoperativer Symptome, der kognitiven und physischen Leistungsfähigkeit sowie der Ernährungssituation wurden die beiden validierten Fragebögen QLQ-C30 und QLQ-STO22 der European Organisation for Research and Treatment of Cancer angewendet.

\section{Ergebnisse}

Von insgesamt 261 operierten Patienten waren noch 91 Patienten am Leben, davon beantworteten 53 ( $\mathrm{TG}$ n=25; SG n=28) die Fragebögen. Es zeigte sich ein prozentual besserer allgemeiner Gesundheitszustand („global health“) in der SG-Gruppe. Patienten in der TG-Gruppe hatten deutlich häufiger postoperative Symptome („higher symptom score“) als Patienten in der SG-Gruppe. Statistisch ausgewertet zeigte sich jedoch kein signifikanter Unterschied in allen gemessenen Parametern. Ausnahme ist eine signifikant erhöhte Dysphagierate in der TG-Gruppe.

\section{Diskussion}

Die vorliegende Studie verdeutlicht erneut, dass nicht nur das onkologische Langzeitergebnis, sondern auch die postoperative Lebensqualität der betroffenen Patienten im gesamten Behandlungskonzept berücksichtigt werden muss. Die Autoren begründen die fehlende Signifikanz ihrer Ergebnisse vor allem mit der geringen Fallzahl und verweisen diesbezüglich auf andere Studien, in denen ein signifikanter
Vorteil der SG gegenüber der TG hinsichtlich der QOL gezeigt werden konnte.

Die interessante und wichtige Fragestellung der Studie kann leider nicht über die erheblichen methodischen Schwächen hinwegtäuschen und somit auch nicht hinreichend beantwortet werden. Vor allem die fehlende Berücksichtigung der unterschiedlichen Tumorstadien und histologischen Subtypen verbietet eine valide Aussage über die Beziehung des Resektionsausmaßes zur QOL. Denn wie bereits in vielen Studien gezeigt werden konnte, ist das Tumorstadium der wichtigste unabhängige Parameter für die QOL nach Magenresektionen. Letztendlich scheint die Durchführung einer SG unter Berücksichtigung der onkologischen Radikalität im Hinblick auf die QOL auf jeden Fall nicht von Nachteil für die betroffenen Patienten zu sein. Unabhängig von dieser Studie zeigt die allgemeine klinische Erfahrung, dass das Resektionsausmaß bei Langzeitüberlebenden hinsichtlich der QOL eher nachrangig ist. In Anbetracht der klaren Definitionen zur Durchführung einer SG bzw. einer TG sollte das Ziel einer R0-Resektion im Vordergrund stehen.

\section{Korrespondenzadresse}

\section{Dr. T. Franzke}

Klinik für Allgemein- und Viszeralchirurgie, Schwerpunkt für endokrine und onkologische Chirurgie, Sektion für Adipositaschirurgie, Diakoniekrankenhaus Henriettenstiftung Hannover,

Marienstr. 72-90, 30171 Hannover

Timm.Franzke@ddh-gruppe.de

Interessenkonflikt. T. Franzke und J. Jähne geben an, dass kein Interessenkonflikt besteht. 